FORMATION Formation emploi

Revue française de sciences sociales

146 | Avril-Juin 2019

L'apprentissage en Allemagne face à ses défis

\title{
Former des apprentis en entreprise : un enjeu de responsabilité sociale?
}

Firms' motivation to train apprentices - A matter of social responsibility?

Betriebliche Ausbildungsmotivation - Eine Frage der gesellschaftlichen

Verantwortung?

Formar aprendices en la empresa: ¿una apuesta de responsabilidad social?

Harald Pfeifer, Gudrun Schönfeld et Felix Wenzelmann

\section{OpenEdition}

Journals

Édition électronique

URL : http://journals.openedition.org/formationemploi/7332

DOI : 10.4000/formationemploi.7332

ISSN : 2107-0946

Éditeur

La Documentation française

Édition imprimée

Date de publication : 26 juin 2019

Pagination : $29-52$

ISSN : 0759-6340

Référence électronique

Harald Pfeifer, Gudrun Schönfeld et Felix Wenzelmann, « Former des apprentis en entreprise : un enjeu de responsabilité sociale? », Formation emploi [En ligne], 146 | Avril-Juin 2019, mis en ligne le 01 janvier 2021, consulté le 07 janvier 2021. URL : http://journals.openedition.org/formationemploi/7332 ; DOI : https://doi.org/10.4000/formationemploi.7332 


\section{Former des apprentis en entreprise : un enjeu de responsabilité sociale?}

Harald Pfeifer

Directeur du département économie de l'EFP (Éducation et formation professionnelle) de I'Institut fédéral pour la formation professionnelle (BIBB - Bundesinstitut für Berufsbildung)

GUDRUN SCHÖNFELD Chercheuse, département économie de l'EFP (Éducation et formation professionnelle) de I'Institut fédéral pour la formation professionnelle (BIBB - Bundesinstitut für Berufsbildung)

Felix WenZeLmann

Chercheur, département économie de l'EFP (Éducation et formation professionnelle) de I'Institut fédéral pour la formation professionnelle (BIBB - Bundesinstitut für Berufsbildung)

Résumé

Former des apprentis en entreprise : un enjeu de responsabilité sociale?

Le système allemand de formation en apprentissage est tributaire de la volonté des entreprises de former les jeunes. L'article examine les motivations qui incitent les entreprises à s'investir dans la formation des apprentis. L'analyse se centre sur les entreprises non concernées par l'argument économique classique mettant en avant la production ou l'investissement comme motivations. Ces entreprises acceptent le coût (net) de formation, bien qu'elles ne souhaitent pas employer leurs apprentis sur des postes d'ouvriers qualifiés une fois l'apprentissage terminé. Cet article émet l'hypothèse que la culture d'entreprise et la responsabilité sociale des entreprises ont un poids relativement plus fort pour cette catégorie d'entreprises. En outre, le segment des entreprises formatrices ayant des motivations non classiques se réduit entre les deux enquêtes mobilisées.

Mots-clés : apprentissage, culture d'entreprise, entreprise formatrice, responsabilité sociale des entreprises, théorie du capital humain, Allemagne

Abstract

Firms' motivation to train apprentices - A matter of social responsibility?

The German apprenticeship training system depends on firms' willingness to train youth. Using German firm-level data, this paper discusses which motives drive firms' participation in training apprentices. A focus is given to those firms that do not fit the standard economic argumentation of the production or investment motive. These firms accept (net) costs of training, although they are not interested in employing their apprentices as skilled workers afterwards. This paper suggest that firm tradition and the social responsi- 
bility of firms are relatively more important in this group of firms. Moreover, the segment of firms not training according to standard motives shrinks over the years between the two reference surveys.

Keywords: apprenticeship, corporate culture, company that provides training, corporate social responsibility, human capital theory, Germany

Journal of Economic Literature: J 24 ; M 51 ; M 54

Traduction : Auteur.e.s

\section{Introduction : pourquoi les entreprises forment-elles des apprentis ?}

Le système allemand d'apprentissage alliant l'apprentissage sur le lieu de travail à l'apprentissage en milieu scolaire suscite toujours un vif intérêt chez les acteurs, qui y voient un moyen de répondre au fort taux de chômage des jeunes dans leurs pays respectifs. Les pays du Sud de l'Europe cherchent à accroître l'apprentissage sur le lieu de travail afin de faciliter la transition entre le monde de l'éducation et le marché du travail, notamment suite à la dernière crise économique.

Une question essentielle préoccupe les partisans de cette approche : comment convaincre les entreprises de participer à la formation des jeunes ? Les entreprises ont tendance à se focaliser sur le coût de la formation sans en reconnaître les intérêts potentiels. Ceci vaut tout particulièrement pour les pays où les entreprises n'ont pas pour tradition de participer à la formation des jeunes et où les structures d'appui institutionnelles sont rares.

L'alternance est au coeur du système d'apprentissage allemand. Les apprentis sont formés à la fois dans des écoles professionnelles bénéficiant de financements publics (un à deux jours par semaine) et dans l'entreprise formatrice avec lesquelles ils signent un contrat d'apprentissage contraignant. En signant ce contrat, les entreprises s'engagent à délivrer la formation nécessaire, conformément au règlement d'apprentissage de la profession concernée ("Ausbildungsordnung"). D’un côté, l'entreprise verse une rémunération à l'apprenti et finance la formation en entreprise. De l'autre, les apprentis participent au processus de production durant leur temps de formation en entreprise, et génèrent donc des avantages pour l'entreprise (pour une vue d'ensemble du système allemand d'apprentissage, voir, par exemple, Fürstenau \& al. (2014) et pour une brève introduction au modèle de calcul des coûts nets de formation, voir encadré $\mathbf{1}$ ).

De nombreux travaux de référence ont tenté d'expliquer pourquoi certaines entreprises s'impliquaient dans l'apprentissage en alternance et pas d'autres (par exemple, Acemoglu \& Pischke, 1998 ; Wolter \& al., 2006 ; Mohrenweiser \& Zwick, 2009). Dans le cas allemand, la participation à la formation en apprentissage s'appuie sur le volontariat. Elle obéit aux principes du marché, à savoir que les entreprises sont libres 
de décider de former, ou pas, des apprentis, mais aussi de leur nombre. Du côté de l'offre, les diplômés de l'enseignement secondaire postulent aux places d'apprentissage disponibles et les entreprises mettent en ouvre un processus de sélection et de recrutement afin de choisir leurs apprentis (Wenzelmann \& al., 2017).

Les motivations les plus courantes des entreprises qui forment des apprentis sont "l'investissement » et la " production ». Afin d'opérer un classement des entreprises selon leur motivation à former, les travaux empiriques mobilisent fréquemment des indicateurs tels que les coûts de formation pour l'entreprise et/ou la stratégie de rétention (Mohrenweiser \& Backes-Gellner, 2010 ; Wenzelmann, 2012 ; Mohrenweiser, 2016). Appliquée à l'Allemagne, cette méthode révèle que la plupart des entreprises formatrices sont prêtes à consacrer des moyens importants à la formation de leurs apprentis (par exemple, Schönfeld \& al., 2016) ; leur motivation relève donc de «l'investissement ». Ces entreprises sont nombreuses à employer leurs anciens apprentis à des postes de travailleurs qualifiés une fois qu'ils ont terminé leur apprentissage et réussi leur examen. Cependant, pour environ un tiers des entreprises formatrices, les coûts de formation sont déjà couverts durant l'apprentissage, car pendant la formation, la productivité des apprentis est supérieure à leur coût à l'issue de l'apprentissage. Pour ces entreprises, la formation répond donc à une " motivation de production ".

Si ces motivations à former des apprentis ont déjà fait l'objet d'analyses approfondies dans la littérature existante, rares sont les travaux qui se sont intéressés au fait que certaines entreprises investissent dans la formation sans intention d'embaucher les apprentis. Le présent article examine les raisons possibles d'un tel positionnement qui, à première vue, ne semble pas justifié d'un point de vue économique.

Dans un premier temps, l'article aborde les motivations des entreprises formatrices en général. Ensuite, les données de deux études allemandes menées en entreprise, sur les coûts et les bénéfices de la formation en apprentissage, sont mobilisées afin de prouver empiriquement le poids des différentes motivations. Pour ce faire, nous mesurons les coûts (nets) de formation, ainsi que les stratégies de rétention des entreprises, car ces deux mesures déterminent les motivations à former. L'étude accorde une attention particulière au groupe d'entreprises formatrices qui présentent des coûts de formation nets positifs, mais n'aspirent pas à retenir les apprentis. Les résultats de notre étude laissent penser que chez ce groupe d'entreprises, plus que parmi les autres, la formation répond à une motivation sociale. Nous observons également que l'importance relative de la motivation sociale diminue au fil du temps entre les deux enquêtes (de 2007 à 2012/13), ce qui entraîne d'importantes répercussions politiques. 


\section{Des entreprises motivées pour former? Théorie du capital humain et au-delà}

Selon la théorie classique du capital humain (TCH) de Becker (1964), les entreprises n'ont pas de véritables intérêts à investir dans le capital humain général (transférable) de leurs employés puisque, dans l'hypothèse de marchés parfaits, elles n'ont aucun moyen de récupérer cet investissement. Les investissements en capital humain spécifique (non transférable) peuvent s'avérer avantageux pour l'entreprise qui peut ainsi verser des salaires inférieurs à la productivité des employés formés.

En Allemagne, la formation en apprentissage génère une grande part de capital humain général ou tout du moins propre à un métier, transférable à d'autres entreprises employant des travailleurs au sein des mêmes professions (Pfeifer \& al., 2011). Les programmes de formation et les examens finaux, externes et centralisés, déterminent un niveau minimum de compétences et connaissances que les apprentis doivent acquérir pendant la formation. Le capital humain acquis accroît ainsi la productivité dans toutes les entreprises employant des travailleurs formés à un métier donné. Dans cet article, nous soutenons donc que le capital humain acquis, spécifique à une profession, est en principe un capital humain général au sens de Becker (1964).

$\mathrm{Du}$ point de vue d'une $\mathrm{TCH}$ classique, les raisons pour lesquelles les entreprises investissent dans la formation en apprentissage restent donc difficiles à déterminer. Différentes études démontrent que l'hypothèse de marchés parfaits est démentie dans les marchés réels (voir Wolter \& Ryan, 2011, pour une vue d'ensemble des travaux récents sur la formation). Une structure salariale ressérée, découlant de l'application du salaire minimum ou d'une négociation collective, permet aux entreprises de verser des salaires inférieurs à la productivité des apprentis formés. Ainsi, un salaire inférieur à la productivité peut contribuer à refinancer l'investissement en formation (Acemoglu \& Pischke, 1998 ; Acemoglu \& Pischke, 1999a ; Acemoglu \& Pischke, 1999b).

$\mathrm{La} \mathrm{TCH}$ et les travaux récents sur la formation jettent les bases théoriques des différentes possibilités de financement de la formation générale. Une entreprise formatrice $(i)$ dispose de trois options pour retirer un bénéfice de la formation : la contribution des apprentis à la production $(P R)$; les bénéfices découlant de la rétention de l'apprenti par l'entreprise $(B R)$; les bénéfices engendrés par la participation au système en tant que tel $(I)$, par exemple en véhiculant une meilleure image de l'entreprise et en envoyant un signal positif en termes de conditions de travail. Une entreprise ne devrait donc former des apprentis que si la somme des bénéfices est au moins égale aux coûts bruts escomptés $(G C)$ de la formation $\left.E\left[G C_{i}\right] \leq E\left[P R_{i}\right]+E\left[B R_{i}\right]+E\left[I_{i}\right]\right)$.

En s'appuyant sur les constats théoriques et empiriques décrits, les économistes font souvent la distinction entre deux stratégies de formation des entreprises. La moti- 
vation de production (Lindley, 1975) est fondée sur les contributions productives des apprentis. Les entreprises ne cherchent pas à retenir les apprentis $(\mathrm{E}[B R]=0)$. En supposant que la valeur $\mathrm{E}[I]$ est constante pour chaque entreprise formatrice, la seule possibilité pour l'entreprise d'améliorer son rapport coût-bénéfice est de réduire les coûts ou d'augmenter la durée de productivité des apprentis.

Si une entreprise cherche principalement à former de futurs travailleurs qualifiés, elle a une motivation d'investissement (Merrilees, 1983). Ainsi, les entreprises mettent l'accent sur les bénéfices escomptés à travers la rétention de leurs anciens apprentis $(\mathrm{E}[B R])$. Les bénéfices escomptés de cette rétention dépendent de trois facteurs : (a) la probabilité qu'un diplômé accepte un contrat de travailleur qualifié ; (b) les compétences acquises pendant la formation ; et (c) la disponibilité d'une main-d'oeuvre de remplacement sur le marché du travail externe. Les entreprises peuvent au moins en partie influencer les facteurs (a) et (b) en offrant des formations de qualité élevée et en créant un environnement de travail positif. Le facteur (c) est exogène, en supposant que les entreprises ne déplacent pas constamment leurs sites de production en fonction de la disponibilité régionale de travailleurs qualifiés.

Il existe une autre variante de la motivation d'investissement, lorsque les entreprises tirent un bénéfice de l'existence d'un vivier de travailleurs qualifiés, dans le cas où il leur faudrait remplacer des travailleurs ou embaucher des travailleurs supplémentaires suite à une reprise économique. Dans certains secteurs économiques, le maintien d'un "stock" de travailleurs pourrait justifier les coûts de formation, même en l'absence d'une demande actuelle de main-d'œuvre (Backes-Gellner, 1996 ; Pfeifer \& BackesGellner, 2018). Cela pourrait être le cas si le marché des travailleurs qualifiés, dans une profession donnée, était tendu et que la survie de l'entreprise dépendait fondamentalement de la disponibilité immédiate de travailleurs qualifiés. Stevens (1994) et Franz \& Zimmermann (2002) examinent le modèle dit " de sélection ». Selon ce modèle, les entreprises utilisent les trois années de formation à titre de période d'essai prolongée, afin de vérifier si l'apprenti convient à l'emploi et à l'entreprise. Étant donné que le modèle de sélection met également l'accent sur les bénéfices postérieurs à la formation, nous avons regroupé cet aspect avec le bénéfice associé à la rétention $\mathrm{E}[B R]$.

Sadowski (1980) introduit la troisième composante du bénéfice (I). L'auteur soutient que certaines entreprises mettent en place une stratégie de formation afin d'améliorer leur réputation auprès de leurs clients, fournisseurs et employés. Comme souligné plus haut, dans le présent article, nous considérons ce bénéfice comme étant constant d'une entreprise à l'autre.

Les études empiriques (Mohrenweiser \& Zwick, 2009 ; Mohrenweiser \& BackesGellner, 2010 ; Wenzelmann, 2012) utilisent deux mesures pour classer les entreprises en fonction de leur motivation de formation : les coûts nets de formation (Schönfeld $\&$ al., 2016) et la rétention (taux ou stratégie) des apprentis. Des coûts nets négatifs et un taux de rétention faible ou nul traduisent une motivation de production. Des 
coûts et un taux de rétention élevés traduisent une motivation d'investissement. Par ailleurs, certaines entreprises pourraient former des apprentis à la fois avec des coûts nets faibles, voire négatifs, et un taux de rétention élevé. Ces entreprises pourraient alors tirer un double dividende de la formation.

La question des entreprises formatrices dont les coûts nets sont élevés et qui ne montrent pas d'intérêt pour la rétention n'est pas explicitement abordée. Dans la suite de cet article, nous décrivons ces entreprises comme ayant une motivation de formation "floue ". Nous nous sommes plus particulièrement intéressés à cette catégorie d'entreprises, car leur volonté d'investir dans la formation de leurs apprentis semble être motivée par des facteurs autres que purement économiques. La partie suivante décrit les données utilisées pour modéliser les différentes motivations.

\section{Les données et l'approche empirique : enquêtes coûts-bénéfices du BIBB}

Pour notre analyse, nous utilisons des données recueillies par l'Institut fédéral pour la formation (BIBB), qui a mené plusieurs enquêtes mesurant les coûts et bénéfices de la formation en apprentissage du point de vue des entreprises. Nous utilisons ici les données des deux dernières enquêtes sur les coûts-bénéfices du BIBB (CBS - Cost-BenefitSurveys) portant sur les années 2007 (Schönfeld \& al., 2010) et 2012/13 (Schönfeld \& al., 2016).

Pour chacune des enquêtes, des échantillons d'adresses sont issus du registre administratif des entreprises de l'Agence fédérale pour l'emploi (BA). Il répertorie toutes les entreprises qui, en Allemagne, emploient au moins un travailleur ou apprenti assujetti aux cotisations de sécurité sociale.

Les entretiens ont été menés avec la personne responsable de la formation, dans la profession choisie et au sein de chaque entreprise, généralement le chef d'entreprise dans les petites entreprises. Il s'agit d'entretiens individuels et assistés par ordinateur (IPAO) d'une durée moyenne de 70 minutes. Le questionnaire comporte des questions très détaillées sur tous les coûts liés à la formation des apprentis dans l'entreprise, qui sont ensuite intégrés dans un modèle de coûts (voir encadré 1). Par ailleurs, les enquêtes ont porté sur les bénéfices et l'organisation de la formation, les caractéristiques de l'entreprise et l'attitude générale à l'égard de la formation en apprentissage.

La série de données de l'enquête de 2007 comporte 2986 entreprises et celle de 2012 2013 en compte 3 032. Aux fins d'analyse avec les données regroupées, nous excluons toutes les entreprises pour lesquelles il manque des valeurs dans l'une des variables 
d'intérêt. La série de données finale comprend ainsi 5675 entreprises (2 769 pour l'enquête de 2007 et 2906 pour celle de 2012-2013).

Comme l'ilustre le tableau 1, les principales caractéristiques des échantillons, pour les différentes années de référence, sont rigoureusement similaires en termes de taille d'entreprise, région et nombre moyen d'apprentis dans les entreprises.

Tableau 1. Résultats descriptifs de l'échantillon groupé

\begin{tabular}{lccc}
\hline & 2007 & $\mathbf{2 0 1 2} / \mathbf{1 3}$ & Total \\
\hline Taille de l'entreprise (en \%) & 54 & 49 & 52 \\
\hline Moins de 10 employés & 33 & 36 & 35 \\
\hline 10 à 49 employés & 12 & 14 & 13 \\
\hline 50 à 499 employés & 1 & 2 & 1 \\
\hline 500 employés ou plus & 84 & 86 & 85 \\
\hline Région (Allemagne de l'0uest, en \%) & & 44 & 46 \\
\hline Secteur de formation (en \%) & 47 & 36 & 34 \\
\hline Industrie et commerce & 31 & 2 & 3 \\
\hline Artisanat & 3 & 15 & 14 \\
\hline Agriculture & 13 & 2 & 4 \\
\hline Professions libérales & 5 & 1 & 0 \\
\hline Fonction publique & - & 2 & 2 \\
\hline Économie domestique & 2 & 2906 & 5675 \\
\hline Nombre d'apprentis & 2769 & & \\
\hline Observations & & 4 & \\
\hline
\end{tabular}

Exemple de lecture : dans l'échantillon de 2007, $54 \%$ des entreprises formatrices ont moins de 10 employés, $33 \%$ ont 10 à 49 employés, $12 \%$ ont 50 à 499 employés et $1 \%$ ont 500 employés ou plus.

Source : BIBB-CBS 2007 et 2012/13. 


\section{Encadré 1. Calcul des coûts nets}

Les coûts nets (NC) sont calculés en différenciant les coûts bruts (GC) et les contributions productives $(P R)$ des apprentis (voir Schönfeld \& al., 2016) :

$$
N C_{i}=G C_{i}-P R_{i}
$$

Les coûts bruts correspondent à la somme des coûts associés aux apprentis ( $C^{\mathrm{app}}$, en particulier l'allocation de formation), aux formateurs ( $\left.\mathrm{C}^{\mathrm{tr}}\right)$, au matériel et à l'infrastructure (Cmat), et des coûts divers ( $\left.C^{\text {oth }}\right)$ :

$$
G C_{i}=C_{i}^{a p p}+C_{i}^{t r}+C_{i}^{\text {mat }}+C_{i}^{o t h}
$$

Les coûts des formateurs $\left(\mathrm{C}^{\text {tr }}\right)$ sont calculés en pondérant le temps que les formateurs passent avec les apprentis par les coûts salariaux des formateurs de chaque groupe. Nous distinguons ici entre les formateurs à temps plein et ceux à temps partiel qui exercent en parallèle de leur emploi d'origine ou qui interrompent ce travail pour former des apprentis. Le temps de formation et de travail parallèle n'est inclus dans le calcul des coûts que dans la mesure où la productivité est réduite. Les formateurs sont souvent aussi productifs pendant les heures de formation qu'en dehors, étant donné qu'ils poursuivent leur travail habituel tout en fournissant des explications. Les frais de recrutement des apprentis ou les coûts d'administration de l'apprentissage sont des exemples de coûts divers.

Pendant leur apprentissage, les apprentis participent généralement au processus de production de l'entreprise, en efffectuant des tâches qualifiées ou non qualifiées. Sans eux, les premières devraient être assurées par des travailleurs non qualifiés et les dernières par des travailleurs déjà formés. Le temps que les apprentis consacrent à des tâches de production non qualifiées ( $h^{\text {us }}$ ou qualifiées $\left(h^{s}\right)$ est utilisé pour calculer les contributions productives $(P R)$. Les tâches non qualifiées sont pondérées par les coûts salariaux des travailleurs non qualifiés $\left(w^{\text {us }}\right)$. Les tâches qualifiées sont pondérées par les coûts salariaux des travailleurs qualifiés $\left(w^{5}\right)$. Toutefois, les apprentis pouvant être moins productifs que les travailleurs déjà formés, nous utilisons un facteur (a) qui mesure la productivité relative des apprentis par rapport à celle du travailleur qualifié moyen, pendant l'exécution de tâches qualifiées, afin de calculer les contributions productives des apprentis.

$$
P R_{i}=h_{i}^{u s} w_{i}^{u s}+\alpha_{i} h_{i}^{s} w_{i}^{s}
$$

Toutes les mesures (a inclus) sont recueillies pendant les entretiens dans chaque entreprise $i$. Jansen \& al. (2015) fournissent une vue d'ensemble de ces chiffres pour l'enquête de 2012/13.

Comme indiqué, nous utilisons les coûts nets de formation (voir encadré 1) et la stratégie de rétention des entreprises pour déterminer la motivation de formation de chacune d'entre elles.

S’agissant de la stratégie de rétention, nous avons utilisé une mesure directe du BIBB-CBS. Il a été demandé aux entreprises d'identifier leur stratégie générale de rétention des apprentis. Les réponses possibles étaient (a) rétention de tous les apprentis, (b) rétention d'une partie des apprentis, ou (c) rétention des apprentis seulement dans des cas exceptionnels. Pour notre analyse, nous définissons les deux premières catégories de réponse comme celles des entreprises ayant un intérêt à pratiquer la rétention. Afin de vérifier la fiabilité de nos résultats, nous excluons le groupe intermédiaire (rétention d'une partie des 
apprentis), étant donné qu'un faible taux de rétention n’explique pas nécessairement un investissement conséquent dans la formation. Pour une autre spécification, nous utilisons le taux de rétention réel pour les trois dernières années au lieu de la stratégie de rétention (voir en annexe de la version électronique). Bien que cette approche présente certains inconvénients ${ }^{1}$, les résultats restent relativement fiables.

La combinaison des coûts nets d'une entreprise et de sa stratégie de rétention se traduit par quatre motivations possibles de formation, présentées dans le tableau $\mathbf{2}$. Les entreprises ayant des coûts nets pendant la formation et montrant un intérêt pour la rétention ont une " motivation d'investissement " (en haut à gauche). Les entreprises n'ayant pas de coûts nets et témoignant d'un intérêt pour la rétention ont une " motivation de double dividende " (en bas à gauche). Les entreprises n'ayant pas de coûts nets et ne montrant pas d'intérêt pour la rétention ont une "motivation de production" (en bas à droite). Enfin, les entreprises ayant des coûts nets et pas d'intérêt pour la rétention ont une " motivation floue " (en haut à droite).

Tableau 2. Motivations de formation des entreprises en fonction des coûts (nets) et de la stratégie de rétention

\begin{tabular}{|c|c|c|c|}
\hline & & \multicolumn{2}{|c|}{ Stratégie de rétention } \\
\hline & & Intérêt de rétention & Pas d'intérêt de rétention \\
\hline \multirow{2}{*}{$\begin{array}{l}\text { Coûts nets de } \\
\text { formation }\end{array}$} & Coûts nets $\left(\mathrm{CN}_{i}>0\right)$ & Motivation d'investissement & Motivation floue \\
\hline & Bénéfices nets $\left(\mathrm{CN}_{\mathrm{j}}<0\right)$ & Motivation de double dividende & Motivation de production \\
\hline
\end{tabular}

Source : auteurs.

Le tableau 2 montre les deux motivations « classiques " décrites, d'investissement et de production. Il présente également la motivation combinant les deux motivations classiques (motivation de double dividende) et la motivation ne correspondant pas exactement aux classifications établies dans la littérature (motivation floue).

Dans le cas d'une motivation floue, l'entreprise accepte des coûts nets malgré son manque d'intérêt à retenir les apprentis. Cela peut être le cas si les coûts réels sont plus élevés qu’initialement prévu ou si l'entreprise forme des apprentis pour des raisons autres

1. Par exemple, nous ne pouvons utiliser qu'un échantillon réduit, car environ $17 \%$ des entreprises interrogées n'avaient pas accueilli de diplômés au cours des trois dernières années. En outre, le taux de rétention réel n'est pas uniquement influencé par l'entreprise elle-même, mais également par les apprentis, qui peuvent être amenés à quitter volontairement l'entreprise, et par d'autres entreprises qui peuvent débaucher les apprentis (Mohrenweiser \& al., 2019). 
qu'économiques, comme la responsabilité sociale ou une tradition d'entreprise (Beicht \&al., 2004). ${ }^{2}$

Dans l'analyse empirique qui suit, nous examinons si les facteurs non économiques (c'est-à-dire la responsabilité sociale ou la tradition d'entreprise) sont relativement plus importants dans le segment des entreprises formatrices dont la motivation est floue et relativement moins importants pour celles dont la motivation de formation est la production ou l'investissement.

Notre analyse empirique commence par une description de la répartition des entreprises selon les quatre quadrants de la motivation de formation. Nous présentons ensuite la répartition en fonction de différentes caractéristiques de l'entreprise, telles que la taille, le secteur de formation ${ }^{3}$ ou le type de profession (commerciale, industrielle, technique $)^{4}$. Dans la deuxième étape, nous utilisons plusieurs modèles de régression multivariée pour analyser la relation entre les variables d'intérêt et la position dans l'un des quadrants. Les modèles contrôlent l'année d'enquête, la taille de l'entreprise, le secteur économique, le type de profession, la région, le type d'entreprise, les conditions du marché du travail, le parcours scolaire des apprentis et les institutions au sein de l'entreprise (par exemple, la présence d'un comité d'entreprise).

\section{Résultats : tradition d'entreprise 3 et responsabilité sociale jouent un rôle important}

Nous présentons ici, dans un premier temps, des résultats descriptifs pour les deux échantillons séparément. Nous prenons en compte les autres motivations à former des apprentis et le degré d'assentiment des entreprises vis-à-vis de ces motifs. Nous présentons ensuite les résultats des régressions logistiques pour chacune des quatre motivations de formation présentées dans le tableau 2, afin de tenir compte de diverses variables supplémentaires.

2. Ceci pourrait être expliqué par le fait que les entreprises de ce panel tirent profit d'une meilleure image $(I)$. Au moins dans l'enquête de 2007, les entreprises ayant une motivation floue revendiquent moins souvent la notion d'image comme un facteur important dans leur décision de formation que les entreprises affichant l'une des autres motivations (cette variable n'est pas incluse dans l'enquête de 2012/13).

3. En Allemagne, chaque secteur de formation est responsable de plusieurs formations professionnelles. Les examens finaux sont, par exemple, organisés par les chambres locales de chaque secteur. Les secteurs de l'industrie et du commerce, ainsi que de l'artisanat, sont ceux qui comptent le plus grand nombre de professions et d'apprentis. Nous utilisons les secteurs de formation plutôt que les secteurs économiques.

4. En raison du grand nombre de formations professionnelles, la taille de l'échantillon ne permet pas d'effectuer des analyses spécifiques par profession. 


\subsection{Résultats descriptifs : $13 \%$ des entreprises ont une motivation de formation floue}

Les résultats descriptifs ${ }^{5}$ du tableau 3 révèlent que, dans les deux enquêtes, une majorité d'entreprises a une motivation d'investissement en matière de formation. Cette part a considérablement augmenté, passant de $55 \%$ en 2007, à $64 \%$ en 2012-2013. La part des trois autres groupes est passée de $9 \%$ à $6 \%$ pour la motivation de production, de $21 \%$ à $19 \%$ pour la motivation de double dividende, et de $15 \%$ à $11 \%$ pour la motivation floue 6 .

S'agissant de la taille de l'entreprise, nous constatons que les petites entreprises se situent plus souvent dans le quadrant de la motivation floue ou de production que les grandes entreprises. Cette différence pourrait s'expliquer, en partie, par le fait que les petites entreprises sont souvent moins intéressées par la rétention de leurs apprentis. Si l'on considère qu'en général leur demande de travailleurs qualifiés est plus faible et qu'elles ont donc moins souvent de postes vacants à pourvoir, l'observation semble plausible. Il se pourrait également que les petites entreprises s'attendent à ne pas réussir à convaincre leurs apprentis de rester dans l'entreprise et qu'elles adaptent leur stratégie de formation et de rétention en conséquence. Néanmoins, la part des entreprises de moins de 10 salariés dont la motivation est floue est étonnamment élevée (plus de $17 \%$ ).

Il est moins surprenant de constater que les grandes entreprises forment plus souvent des apprentis pour disposer d'une main-d'oeuvre qualifiée pour le futur. Toutefois, la part des entreprises qui forment des apprentis sans coûts nets et qui, dans le même temps, ont l'intention de conserver au moins une partie de leurs apprentis, est supérieure à $22 \%$ pour les entreprises de plus de 10 salariés. D'une part, ces entreprises évitent le risque consistant à engager des coûts irrécupérables dans le cas où les apprentis seraient débauchés par une autre entreprise ou démissionneraient pour d'autres raisons. D'autre part, la formation intervenant dans le processus de production est considérée comme une formation de qualité élevée dans la littérature sur l'éducation. Des coûts nets faibles ou nuls représentent donc un effet secondaire positif de la décision pédagogique d'intégrer la formation dans le processus de travail.

Quant aux différents secteurs économiques (de formation), celui de "l'industrie et du commerce " affiche la plus faible proportion d'entreprises non disposées à embaucher les apprentis. En moyenne, les coûts nets de formation sont relativement élevés dans ce secteur (Schönfeld \& al., 2016), car les professions concernées sont souvent plus complexes ou exigent plus de pratique avant que les apprentis puissent être productifs.

5. Tous les résultats descriptifs sont pondérés pour s'assurer qu’ils ne sont pas faussés du fait de la surreprésentation de certains types d'entreprises dans l'échantillon.

6. À l'exception du double dividende, tous les changements sont significatifs à $1 \%$. 
Toutefois, environ $21 \%$ des entreprises de ce secteur se situent dans le quadrant du double dividende. La part des entreprises dont la motivation est floue est la plus faible de ce secteur (10\%). Dans le secteur de "l'artisanat", la part des entreprises dont la motivation est floue est légèrement plus élevée, tandis que la part de celles qui affichent une motivation d'investissement est plus faible. Le poids des deux autres motivations est comparable à celui observé pour " I industrie et le commerce ". Dans le secteur de la "fonction publique ", les parts sont semblables à celles du secteur de "l'artisanat». La répartition des entreprises du secteur de "l'agriculture " entre les quatre motivations est sensiblement la même, avec toutefois la plus faible proportion d'entreprises ayant une motivation d'investissement (23\%). Dans le secteur des "professions libérales " ${ }^{7}$ la part des entreprises ayant une motivation d'investissement est également relativement faible (49\%), tandis que la part des autres motivations est supérieure à la moyenne.

Par conséquent, dans tous les secteurs et toutes les tailles d'entreprises, une part importante des entreprises forme des apprentis, malgré des coûts nets et une absence d'intérêt à les embaucher après leur apprentissage.

Nous utilisons ensuite les informations issues de nos données, qui évaluent directement la satisfaction des entreprises à l'égard du rapport coût-bénéfice ${ }^{8}$. Il s'agit d'analyser s'il existe des différences entre les entreprises des quatre quadrants. La figure 1 montre que, parmi les entreprises dont la motivation est floue, près d'une sur deux se dit satisfaite ou très satisfaite du rapport coût-bénéfice de la formation au sein de l'entreprise, et seulement $20 \%$ se déclarent insatisfaites. La part des entreprises très satisfaites dans ce groupe a même considérablement augmenté, pour atteindre $18 \%$ en 2012-2013. Parmi les entreprises affichant l'une des autres motivations, plus de $60 \%$ sont satisfaites et seulement $10 \%$ sont insatisfaites. La différence entre les groupes n'est pas aussi marquée qu'on pouvait s'y attendre, compte tenu de leur position dans le tableau des motivations; la plupart des entreprises dont la motivation est floue ne se plaignent pas du rapport coût-bénéfice. La question qui se pose est donc la suivante : quels bénéfices, autres que ceux associés à l'investissement et à la production, pourraient inciter ces entreprises à s'engager davantage dans la formation?

7. Les professions libérales sont par exemple les médecins, les avocats, les notaires.

8. La question peut être formulée ainsi : "Dans l'ensemble, dans quelle mesure êtes-vous satisfait(e) du rapport coût-bénéfice de la formation dans votre entreprise? " 
Tableau 3. Répartition des motivations de formation en \%, selon les caractéristiques de l'entreprise

\begin{tabular}{|c|c|c|c|c|c|}
\hline & $\begin{array}{l}\text { Motivation } \\
\text { floue }\end{array}$ & $\begin{array}{l}\text { Motivation de } \\
\text { production }\end{array}$ & $\begin{array}{c}\text { Motivation } \\
\text { d'investissement }\end{array}$ & $\begin{array}{c}\text { Motivation de } \\
\text { double dividende }\end{array}$ & Total \\
\hline \multicolumn{6}{|l|}{ Année } \\
\hline 2007 & 15 & 9 & 55 & 21 & 49 \\
\hline $2012 / 13$ & 11 & 6 & 64 & 19 & 51 \\
\hline \multicolumn{6}{|l|}{$\begin{array}{l}\text { Taille de } \\
\text { l'entreprise }\end{array}$} \\
\hline 1 à 9 employés & 17 & 9 & 55 & 18 & 52 \\
\hline 10 à 49 employés & 10 & 6 & 63 & 22 & 35 \\
\hline $\begin{array}{l}50 \text { à } \\
499 \text { employés }\end{array}$ & 6 & 3 & 66 & 25 & 13 \\
\hline $\begin{array}{l}500 \text { employés } \\
\text { ou plus }\end{array}$ & 5 & 1 & 72 & 22 & 1 \\
\hline \multicolumn{6}{|l|}{$\begin{array}{l}\text { Secteur de } \\
\text { formation }\end{array}$} \\
\hline $\begin{array}{l}\text { Industrie et } \\
\text { commerce }\end{array}$ & 10 & 6 & 64 & 21 & 46 \\
\hline Artisanat & 15 & 6 & 61 & 19 & 34 \\
\hline Agriculture & 26 & 27 & 23 & 25 & 3 \\
\hline $\begin{array}{l}\text { Professions } \\
\text { libérales }\end{array}$ & 17 & 10 & 49 & 24 & 14 \\
\hline Fonction publique & 16 & 7 & 64 & 14 & 4 \\
\hline \multicolumn{6}{|l|}{ Type de profession } \\
\hline $\begin{array}{l}\text { Profession } \\
\text { commerciale }\end{array}$ & 11 & 7 & 58 & 23 & 44 \\
\hline $\begin{array}{l}\text { Profession } \\
\text { industrielle }\end{array}$ & 16 & 8 & 56 & 19 & 38 \\
\hline $\begin{array}{l}\text { Profession } \\
\text { technique }\end{array}$ & 10 & 4 & 69 & 16 & 17 \\
\hline Total & 13 & 7 & 59 & 20 & 100 \\
\hline
\end{tabular}

Exemple de lecture : en 2007, $15 \%$ des entreprises formatrices ont une motivation de formation floue, $9 \%$ ont une motivation de production, $55 \%$ ont une motivation d'investissement et $21 \%$ ont une motivation de double dividende. $49 \%$ des entreprises font partie de l'échantillon de l'année 2007. Source : BIBB-CBS 2007 et 2012/13; observations : 5675. 
Figure 1. Satisfaction quant au rapport coût-bénéfice de la formation (en \%)

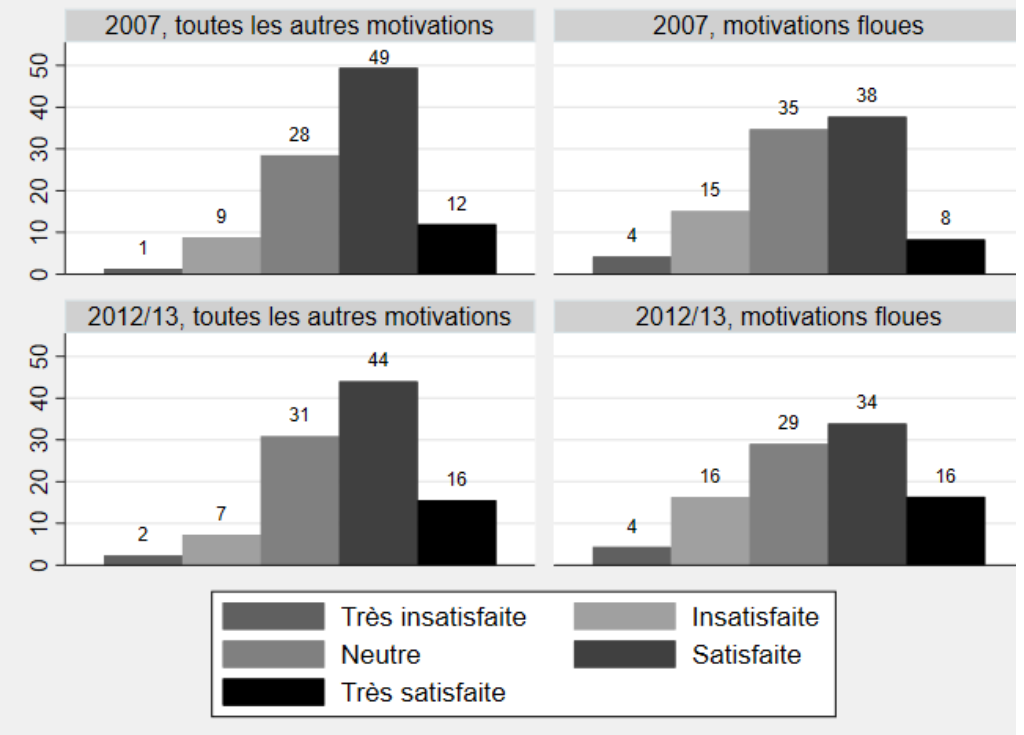

Figure 2. Importance de la motivation « la formation est une tradition d'entreprise » (en \%)

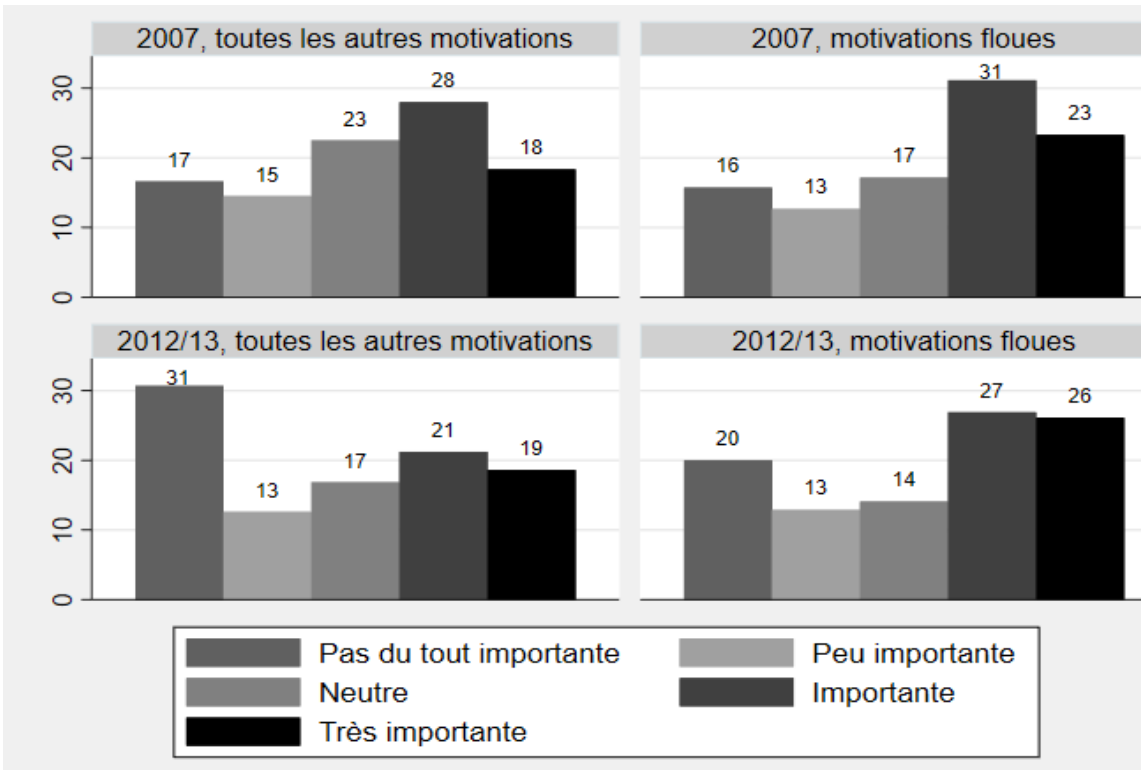

Source : BIBB-CBS 2007 et 2012/13. 
En mobilisant d'autres d'informations issues de nos données sur les raisons de former des apprentis, nous constatons que les arguments non économiques ont plus de poids parmi les entreprises dont la motivation de formation est floue. Quant aux principales raisons pour lesquelles elles forment des apprentis, la figure $\mathbf{2}$ montre que ces entreprises mettent, plus souvent que d'autres, l'accent sur la notion de " tradition d'entreprise ». Parmi les entreprises dont la motivation était floue en 2007, 54 \% affirment que la tradition est un facteur déterminant dans leur décision de formation, contre seulement $46 \%$ des entreprises ayant d'autres motivations. ${ }^{9}$ Dans l'enquête de 2012-2013, la part des entreprises dont la motivation est floue et qui affirment que la tradition d'entreprise est un facteur de formation très important a augmenté, tandis que la part de celles qui affirment que c'est un motif important a diminué presque d'autant. En 2012-2013, pour près d'un tiers des entreprises déclarant l'une des autres motivations, la "tradition d'entreprise " est un motif négligeable dans la formation des apprentis. Cette part a presque doublé par rapport à 2007.

En 2007, 62 \% des entreprises dont la motivation est floue désignent la « responsabilité sociale " comme l'un des facteurs déterminants dans leur décision de formation ; parmi les entreprises indiquant une autre motivation, $59 \%$ se réferent aussi au facteur de responsabilité sociale (figure 3). Cependant, l'enquête la plus récente a montré que $81 \%$ des entreprises ayant une motivation floue affirmaient que la formation représentait, à leurs yeux, une responsabilité sociale. Parmi les entreprises ayant une autre motivation, cette part s'élève à environ $61 \%$, soit un chiffre également relativement élevé. ${ }^{10}$

9. La part de celles qui jugent la tradition importante ou très importante est nettement plus élevée dans le groupe des entreprises dont la motivation est floue.

10. Nous reconnaissons que ce résultat pourrait être biaisé par les attentes sociales, mais nous ne nous atendons pas à un biais différent entre les entreprises ayant différentes motivations de formation. 
Figure 3. Importance de la motivation « la formation est une responsabilité sociale » (en \%)

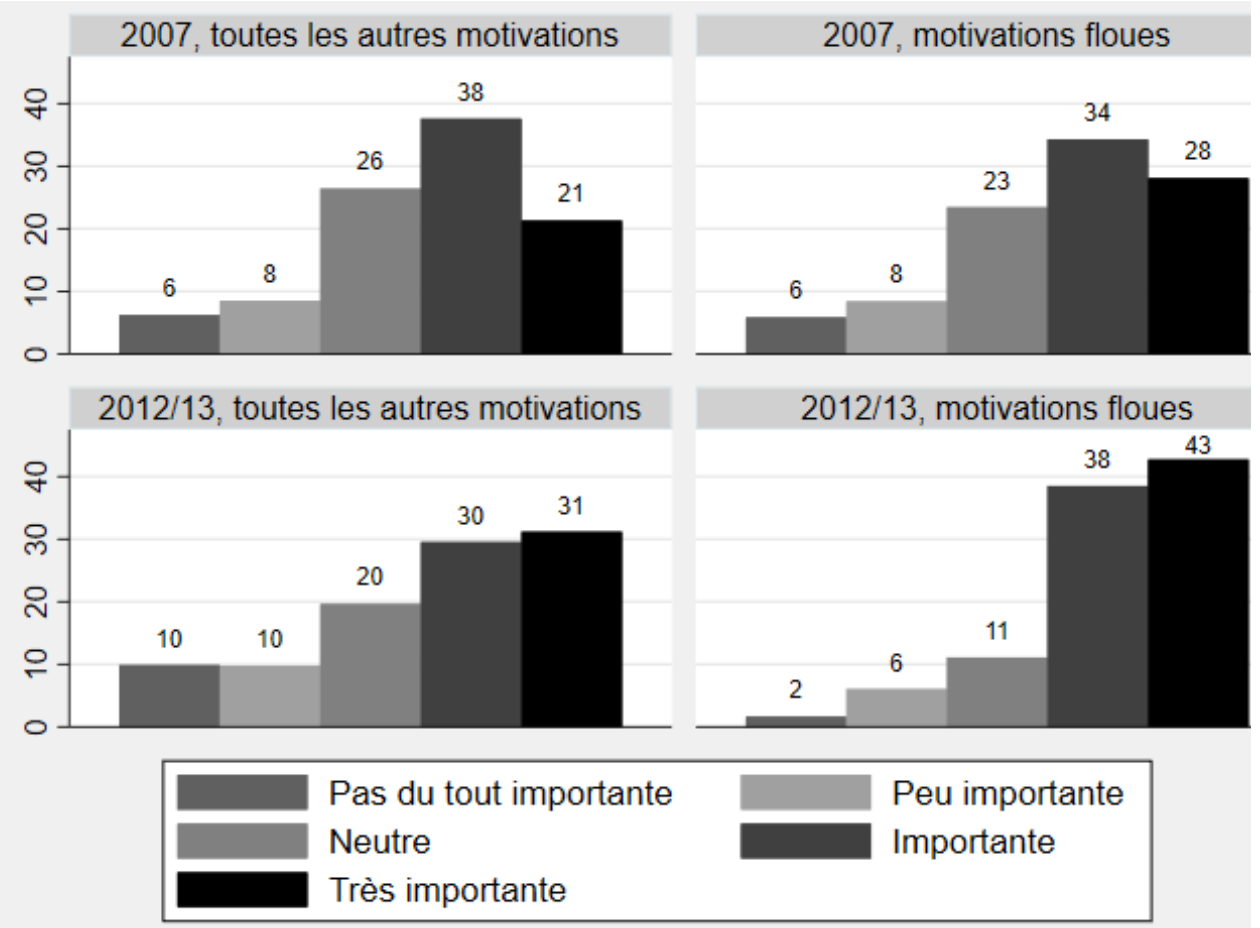

Source : BIBB-CBS 2007 et 2012/13.

Il semble donc qu'une part importante des entreprises qui ne retirent pas un bénéfice économique direct de la formation soient motivées par des facteurs non économiques. La question de savoir si cette observation descriptive reste valide après une analyse de régression tenant compte des variables structurelles est abordée dans la partie suivante. 


\subsection{Analyse de régression : la motivation de formation floue correspond à un motif de responsabilité sociale}

Dans notre analyse multivariée, nous avons appliqué une régression logistique distincte pour chaque quadrant de la matrice des motivations du tableau 2 (motivation d'investissement, motivation de production, motivation de double dividende, motivation floue). ${ }^{11}$ La variable dépendante est égale à un dans le cas où l'entreprise appartient au quadrant concerné et à zéro dans le cas contraire. La figure $\mathbf{4}$ présente les effets marginaux des coefficients et les intervalles de confiance à $95 \%$ d'une sélection de variables indépendantes de la régression des quatre motivations. Le tableau A1, en annexe de la version électronique de l'article, présente les résultats de régression pour toutes les variables indépendantes.

Les points et les lignes correspondantes de la figure 4 représentent les résultats pour les entreprises dont la variable dépendante est une motivation floue. Les losanges représentent une motivation de production, tandis que les carrés font référence à une motivation d'investissement. Enfin, les triangles et les lignes correspondantes montrent les résultats pour les entreprises ayant une motivation de double dividende.

Les coefficients indiquant l'entreprise interrogée en 2012/13 sont négatifs et significatifs dans les régressions des motivations de production, insignifiants pour les motivations floue et de double dividende, et positifs et significatifs pour la motivation d'investissement. La motivation d'investissement a donc progressé, tandis que le nombre d'entreprises ayant une motivation de production a diminué. Les entreprises qui forment aux professions industrielles ou techniques sont plus susceptibles d'avoir une motivation floue et une moindre motivation de double dividende. S'agissant de la taille des entreprises et du secteur de formation, les résultats sont conformes à ceux de l'analyse descriptive. Par exemple, les petites entreprises sont les plus susceptibles d'avoir une motivation floue ou de production.

Les comités d'entreprise sont censés avoir une double influence sur la formation, et donc sur la position dans la matrice de motivation. D'une part, ils sont tenus par la loi de contrôler la qualité de la formation et, d'autre part, ils veillent à ce que l'apprenti ne soit pas utilisé en remplacement des travailleurs réguliers. Ces deux rôles ont un effet positif sur les coûts nets de formation (Kriechel \& al., 2014). En outre, les comités d'entreprise font souvent pression en faveur de l'embauche des apprentis, ce qui pourrait influencer la stratégie de formation de l'entreprise. Les coefficients

11. Les modèles logistiques multinomiaux (mlogit) constituent une méthode alternative d'analyse des données. Dans ces modèles, chaque groupe (ici motivation de formation) est comparé à un groupe de référence. Nous n'avons pas trouvé de résultats divergents en utilisant ce type de modèle. Nous préférons le modèle logistique, étant donné que c'est la décision de former en fonction d'une motivation, par rapport à toutes les autres motivations, qui nous intéresse. 
du comité d'entreprise sont négatifs et significatifs pour les motivations de production et de double dividende, confirmant les relations attendues. Dans les deux autres modèles, ils sont positifs, mais significatifs seulement dans le modèle de la motivation d'investissement.

Si la main-d'oeuvre qualifiée est disponible en abondance sur le marché du travail externe, les entreprises tendent à adopter une approche davantage axée sur la production ou affichent une motivation floue et sont moins souvent tournées vers l'investissement. En cas d'offre excédentaire de travailleurs qualifiés, les avantages de la rétention diminuent, car les travailleurs qualifiés peuvent être recrutés à faible coût. Une stratégie d'investissement est ainsi moins rentable.

Le coefficient positif de l'importance d'une tradition de formation dans la participation d'une entreprise à la formation est significatif à $5 \%$ dans la régression de la motivation floue. Dans les trois autres régressions, il est non significatif.

Comme les résultats descriptifs l'indiquaient déjà, les entreprises qui affirment que la formation relève d'une responsabilité sociale sont plus susceptibles de se trouver dans le quadrant des motivations floue ou de production et moins susceptibles de se trouver dans le quadrant des motivations d'investissement ou de double dividende.

La synthèse des résultats pour le groupe cible de cet article, c'est-à-dire pour les entreprises dont la motivation est floue, suggère que la tradition d'entreprise et la responsabilité sociale jouent un rôle important dans la participation à la formation. 


\section{Figure 4. Effets marginaux des régressions logistiques sur la motivation de} formation ( $\mathrm{N}=5675)$

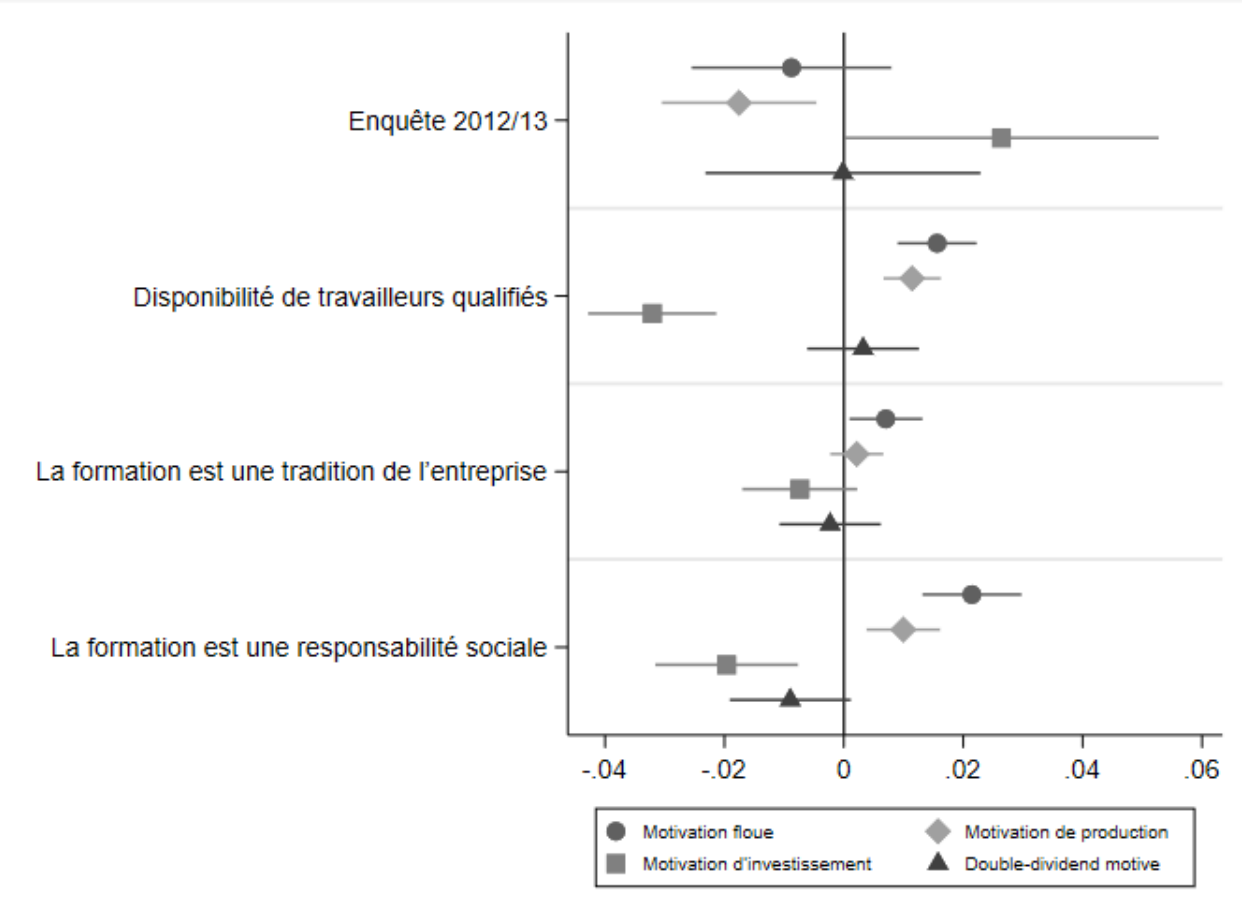

Les effets marginaux moyens sont présentés ici ; nous utilisons des écarts-types robustes.

Variables de contrôle : taille de l'entreprise, région, type d'entreprise, secteur de formation, type de profession, part des apprentis avec Abitur (baccalauréat).

Exemple de lecture: dans la régression logistique pour le groupe dont la motivation est floue (points), l'effet marginal moyen de la variable «l a formation est une responsabilité sociale » a une valeur de 0,02 et est significatif à $5 \%$. Les entreprises qui affirment que la formation relève d'une responsabilité sociale sont donc plus susceptibles d'appartenir au groupe dont la motivation est floue.

Voir le tableau A1 en annexe de la version électronique de l'article pour consulter l'ensemble des résultats.

Source : BIBB-CBS 2007 et 2012/13.

Afin de déterminer si l'importance de la tradition et de la responsabilité sociale chez les entreprises dont la motivation est floue évolue entre les enquêtes, nous avons effectué des régressions distinctes pour chacune des années d'enquête. La figure $\mathbf{5}$ montre que la responsabilité sociale gagne en influence entre les deux enquêtes. Dans la régression de l'enquête de 2007, le coefficient n'est significatif qu'à $10 \%$, alors qu'il est très significatif dans la régression de 2012-2013. Ces résultats n’indiquent pas nécessairement que la responsabilité sociale gagne du terrain en tant que motivation de formation; il se peut également que les entreprises de l'enquête de 2007, dont la motivation de formation n'était pas la responsabilité sociale, soient sorties du quadrant des entreprises à la motivation floue ou ne soient plus des entreprises formatrices dans l'enquête la plus récente. Ainsi, la 
motivation liée à la responsabilité sociale a gagné en importance relative dans le quadrant des motivations floues qui, à son tour, a diminué dans l'absolu (voir le tableau 3). Les implications de ces résultats sont discutées en conclusion.

Figure 5. Effets marginaux des régressions logistiques sur la motivation de formation «floue » 2007 et 2012/13

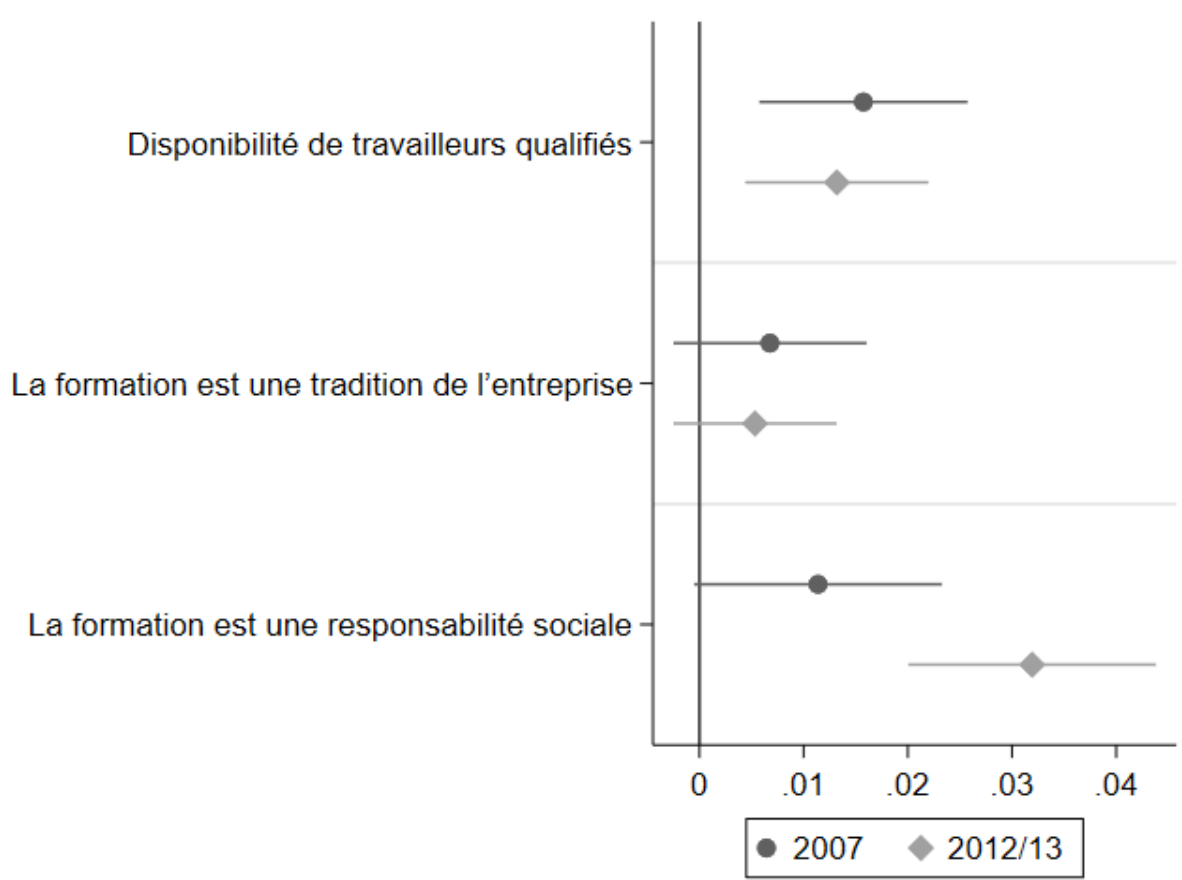

Les effets marginaux moyens sont présentés ici ; nous utilisons des écarts-types robustes.

Variables de contrôle : taille de l'entreprise, région, type d'entreprise, secteur de formation, type de profession, part des apprentis avec Abitur (baccalauréat).

Exemple de lecture : dans la régression logistique pour le groupe dont la motivation est floue pour l'échantillon de 2012/13 (losanges), l'effet marginal moyen de la variable « la formation est une responsabilité sociale » a une valeur de 0,03 et est significatif à $5 \%$. Les entreprises qui affirment que la formation relève d'une responsabilité sociale sont donc plus susceptibles d'appartenir au groupe dont la motivation est floue.

Voir le tableau A2 en annexe de la version électronique de l'article pour consulter l'ensemble des résultats.

Source : BIBB-CBS 2007 et 2012/13. 


\section{Conclusion : pour une meilleure prise en compte, par les décideurs politiques, des différentes motivations de formation}

L'analyse empirique présentée dans cet article met en lumière l'importance relative des motivations de formation des entreprises dans le système d'apprentissage allemand. Les résultats révèlent qu'en dehors des motivations notoires de production et d'investissement, une part significative des entreprises a une motivation de formation floue.

Les résultats de la régression suggèrent que, à l'exception d'autres facteurs potentiels, la responsabilité sociale est un facteur non négligeable pour les entreprises appartenant au quadrant des motivations floues. Cette motivation à former des apprentis gagne même du terrain entre 2007 et 2012/13. Toutefois, l'article révèle également que l'importance relative de la motivation d'investissement progresse, tandis que celle de la motivation floue diminue avec le temps.

D'une part, ces observations laissent supposer que les motivations de formation non économiques deviennent moins fréquentes dans le système d'apprentissage allemand. Cette évolution s'explique, notamment, par le fait que la concurrence (mondiale) croissante et la déréglementation des marchés des produits et du travail peuvent inciter les entreprises à fonder davantage leurs décisions de formation sur des critères économiques (d'efficacité). D'autre part, les résultats suggèrent que la responsabilité sociale reste un facteur non négligeable, pour les entreprises allemandes, dans la formation des apprentis.

Sans juger si cette évolution est socialement souhaitable, il nous semble que les décideurs politiques devraient prendre conscience des conséquences de ces changements dans l'élaboration de leurs stratégies d'éducation et de formation. Compte tenu de la récente baisse de l'engagement des petites entreprises en matière de formation, en Allemagne (Troltsch, 2017), il conviendrait qu'ils évaluent les conditions-cadres existantes et prennent des mesures afin d'inciter plus particulièrement les petites entreprises à former des apprentis pour des raisons économiques. Parmi les mesures proposées par les entreprises elles-mêmes, on peut citer les structures d'appui à la formation, la réduction des obstacles bureaucratiques à la formation et l'assouplissement des modalités de combinaison de l'enseignement professionnel et de la formation en entreprise (Schönfeld \& al., 2016).

Au sein des pays où il n'existe pas de système établi de formation duale ou en situation de travail, les décideurs politiques doivent réaliser que la tradition et la responsabilité sociale sont des motivations potentiellement moins importantes pour la formation. Lorsque la tradition de formation en entreprise n'existe pas par définition ou que l'éducation est considérée comme une responsabilité publique et/ou individuelle, les entreprises ne s'estiment pas responsables de l'investissement dans la formation. Bien que les limites des données ne permettent pas une interprétation causale de nos 
résultats, elles fournissent en revanche un point de départ intéressant pour d'autres recherches dans ce domaine. Il pourrait être intéressant de savoir si les apprentis issus d'entreprises dont la motivation est floue réussissent mieux sur le marché du travail que ceux provenant d'entreprises ayant d'autres motivations.

\section{Bibliographie}

Acemoglu D. \& Pischke J.-S. (1998), "Why do firms train? Theory and evidence", Quarterly Journal of Economics, 113(1), pp. 79-119.

Acemoglu D. \& Pischke J.-S. (1999a), "Beyond Becker: Training in Imperfect Labour Markets", The Economic Journal, 109(453), pp. 112-142.

Acemoglu D. \& Pischke J.-S. (1999b), "The structure of wages and investment in general training", Journal of Political Economy, 107(3), pp. 539-572.

Backes-Gellner U. (1996), Betriebliche Bildungs- und Wettbewerbsstrategien im deutsch-britischen Vergleich: Ein Beitrag der Personalökonomie zur internationalen Betriebswirtschaftslehre, Hampp, München.

Becker G. S. (1964), Human Capital. A theoretical and empirical analysis with special reference to education, Univ. of Chicago Pr., Chicago.

Beicht U., Walden G. \& Herget H. (2004), Kosten und Nutzen der betrieblichen Berufsausbildung in Deutschland, Bertelsmann, Bielefeld.

Franz W. \& Zimmermann V. (2002), "The transition from apprenticeship training to work", International Journal of Manpower, 23(5), pp. 411-425.

Fürstenau B., Pilz M. \& Gonon P. (2014), "The Dual System of Vocational Education and Training in Germany - What Can Be Learnt About Education for (Other) Professions", in Billett S., Harteis C. \& Gruber H. (dir.), International Handbook of Research in Professional and Practice-based Learning. Springer, Dordrecht, pp. 427-460.

Jansen A., Pfeifer H., Schönfeld G. \& Wenzelmann F. (2015), Ausbildung in Deutschland weiterhin investitionsorientiert - Ergebnisse der BIBB-Kosten-NutzenErhebung 2012/13, BIBB Report 1.

Lindley R. M. (1975), "The Demand for Apprentice Recruits by the Engineering Industry, 1951-71", Scottish Journal of Political Economy, 22(1), pp. 1-24.

Merrilees W. J. (1983), "Alternative Models of Apprentice Recruitment: With Special Reference to the British Engineering Industry", Applied Economics, 15(1), pp. 1-21.

Mohrenweiser J. (2016), "Recruitment and apprenticeship training", Industrielle Beziehungen/The German Journal of Industrial Relations, 23(1), pp. 6-24. 
Mohrenweiser J. \& Backes-Gellner U. (2010), "Apprenticeship Training: For Investment or Substitution?" International Journal of Manpower, 31(10), pp. 545-562.

Mohrenweiser J. \& Zwick T. (2009), "Why do firms train apprentices? The net cost puzzle reconsidered", Labour Economics, 16(6), pp. 631-637.

Mohrenweiser J., Zwick T. \& Backes-Gellner U. (2019), "Poaching and FirmSponsored Training", British Journal of Industrial Relations, 57(1), pp. 143-181.

Kriechel B., Mühlemann S., Pfeifer H. \& Schütte M. (2014), "Works Councils, Collective Bargaining, and Apprenticeship Training - Evidence From German Firms", Industrial Relations, 53(2), pp. 199-222.

Pfeifer H. \& Backes-Gellner U. (2018), "Another piece of the puzzle: Firms' investment in training as production of optimal skills inventory", Empirical Research in Vocational Education and Training, 10(6).

Pfeifer H., Schönfeld G. \& Wenzelmann F. (2011), "How large is the firm-specific component of German apprenticeship training?" Empirical Research in Vocational Education and Training, (3)2, pp. 85-104.

Sadowski D. (1980), Berufliche Bildung und betriebliches Bildungsbudget. Zur ökonomischen Theorie der Personalbeschaffungs- und Bildungsplanung im Unternehmen, Poeschel, Stuttgart.

Schönfeld G., Jansen A., Wenzelmann F. \& Pfeifer H. (2016), Kosten und Nutzen der dualen Ausbildung aus Sicht der Betriebe - Ergebnisse der fünften BIBB-KostenNutzen-Erhebung, Bertelsmann, Bielefeld.

Schönfeld G., Wenzelmann F., Dionisius R., Pfeifer H. \& Walden G. (2010), Kosten und Nutzen der dualen Ausbildung aus Sicht der Betriebe. Ergebnisse der vierten BIBB-Kosten-Nutzen-Erhebung, Bertelsmann, Bielefeld.

Stevens M. (1994), "A theoretical model of on-the-job training with imperfect competition", Oxford Economic Papers, 46(4), pp. 537-562.

Troltsch K. (2017), "Betriebliche Ausbildungsbeteiligung - Ergebnisse der Beschäftigungsstatistik zur Ausbildungsbeteiligung", in Bundesinstitut für Berufsbildung (dir.), Datenreport zum Berufsbildungsbericht 2017. Informationen und Analysen zur Entwicklung der beruflichen Bildung, Bertelsmann, Bielefeld, pp. 214-226.

Wenzelmann F. (2012), 'Ausbildungsmotive und die Zeitaufteilung der Auszubildenden im Betrieb', Journal for Labour Market Research, 45(2), pp. 125-145. 
Wenzelmann F., Mühlemann S. \& Pfeifer H. (2017), "The costs of recruiting apprentices: Evidence from German workplace-level data", German Journal of Human Resource Management, 31(2), pp. 108-131.

Wolter S. C., Mühlemann S. \& Schweri J. (2006), "Why Some Firms Train Apprentices and Many Others Do Not", German Economic Review, 7(3), pp. 249-264.

Wolter S. C. \& Ryan P. (2011), "Apprenticeship", in Hanushek E. A., Machin S. \& Wössmann L. (dir.), Handbook of Economics of Education Vol. 3, Elsevier, Amsterdam, pp. 521-576. 